\title{
Conditioned suppression maintained by loud noise instead of shock'
}

\author{
J. F. BRODY, JR. \\ UNIVERSITY OF PITTSBURGH
}

Three Rhesus monkeys were trained on a VI schedule and habituated to the CS (flashing houselights or a clicker). The IIS was $1 \mathrm{sec}$. of $115 \mathrm{db}$ noise presented with CS termination. After pairing with the 'IS the two CS were equally effective in producing response suppression.

Electric shock is customarily the aversive stimulus in punishment, escape, avoidance, and conditioned suppression designs. It is necessary to use a different noxious stimulus in these paradigms before one can be certain that findings are due to shock as a representative aversive source and not to special characteristics of shock. Harrison \& Abelson (1959), Barry \& Harrison (1957), and Harrison \& Tracy (1955) have used noise to maintain escape behavior. However, it was of slower development and lower rates than have been found with shock. Holz \& Azrin (1962) found that pigeons would lower their response rates when punished by $138 \mathrm{db}$ noise but the suppression disappeared with continued training. Azrin (1960) reported similar findings when low intensity shock was used as punishment. Leitenberg (1966) applied the conditioned suppression paradigm to pigeons with $3 \mathrm{sec}$. of intense noise as the US. He found no change in responding that was consistent across sessessions or Ss. The present study also attempted to establish conditioned suppression using high intensity noise as the US.

Method

Three male Rhesus monkeys weighing 18, 16, and 13 lbs. were used. All had previous experience on FR 13 (food reward) while alpha-methyl dopa, alpha-methyl tyrosine, pargyline, or reserpine were administered. No drugs had been given to these animals for at least 18 months prior to the present study.

Training and testing were conducted in a Lehigh Valley Electronics operant conditioning chamber (Model 1469). Standard electro-mechanical circuits controlled the experimental contingencies and data were taken on cumulative recorders and event counters. Simultaneous flashing of the houselights served as one CS. A 4 in. speaker provided the other CS when activated by a Foringer click generator (Model 1293) set at $70 \mathrm{db}$ and 7 cps. The glass panel which was inset into the sliding door of the inner chamber was removed and replaced with a sheet of clear Plexiglas. This Plexiglas pane was drilled with $1 / 16$ th in. holes spaced 1 in. apart, covering an area congruent with a 7 in., 4 ohm speaker which was bolted to the exterior surface of the Plexiglas. The US was delivered to the 7 in. speaker from a Foringer white noise generator (Model 1291), linked with a $15 \mathrm{w}$ public address amplifier. The amplifier was left on continuously and the US was provided by closure of a relay between the amplifier and the speaker. A Dawe sound meter, type $1400 \mathrm{E}$, was used for intensity measurement. Since the operant box had viewing windows it was possible to make intensity determinations with the doors to both cubicles shut.

Session length was $30 \mathrm{~min}$. Water was present ad lib in the operant unit and Ss were kept at normal weight and fed immediately after testing. Ss were trained for 3 months to bar press for $45 \mathrm{mgm}$ dextrose pellets
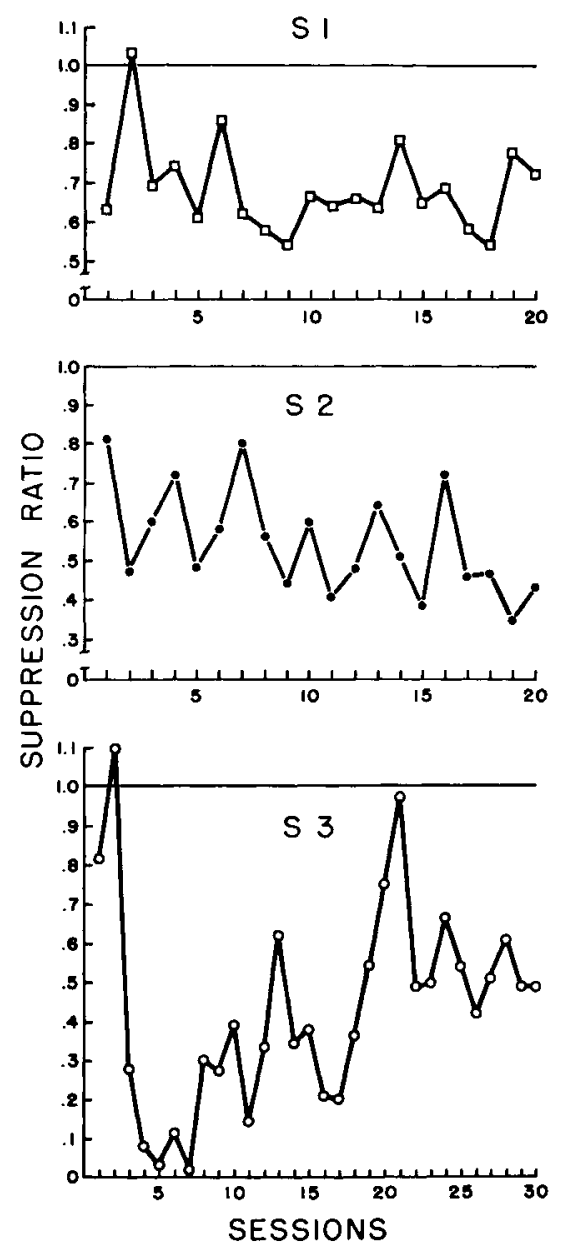

Fig . 1. The mean suppression ratio per session for each $S$. The number of responses emitted during the $\mathrm{CS}$ was divided by the number occurring within $60 \mathrm{sec}$. prior to CS onset. The three ratios obtained for each session were summed and divided by three to produce the session means which are plotted above. 
which were given at a mean interval of $20 \mathrm{sec}$. (VI 20 sec.). In the habituation phase the VI training conditions remained in effect except for CS presentations. CS length was $1 \mathrm{~min}$. and Ss received three CS exposures per session. Intervals between CS were changed daily and varied from 3 to $17 \mathrm{~min}$. The CSs were presented in mixed order with the restriction that Ss would have 10 exposures to each CS by the end of the seventh habituation session. After the habituation phase all CSs were terminated by $1 \mathrm{sec}$. of $115 \mathrm{db}$ noise; measurement indicated no variation greater than $1 \mathrm{db}$ across different portions of the box. S 1 and S 2 were given 60 exposures and S 390 exposures to the US. The extension was made for $S 3$ because of the strong trend toward complete recovery of responding during the CS in sessions 17 through 20.

\section{Results and Discussion}

Figure 1 gives a measure of the suppression produced by the CS in each session. The mean suppression ratios during the last habituation session were $.87, .91$, and .82 for $S 1, S 2$, and $S 3$ respectively. No systematic differences in the degree of suppression produced by the two CSs were observed. There was a pronounced depression of responding during the first $60 \mathrm{sec}$. after each US termination. This depression appeared in all Ss and four to five sessions were required for recovery.

The present study demonstrates that suppression of VI responding can be produced by a CS that has been associated with intense noise. Leitenberg (1966) found suppression similar in degree but less reliable than that reported in this paper. The difference probably relates to the species used, US duration, or US frequency. The parameters controlling the aversive properties of vari- ous stimuli should be explored for several reasons. Intense shock produces severe behavioral disruption. Extreme fear, pain, or postural responses may develop that are relatively insensitive to continued training, schedule changes, or drug effects. Intense noise or low level shock produces moderate response suppression just as the VI schedule maintains a moderate response rate. Correspondingly, behavior controlled by less aversive stimuli may be more sensitive to further experimental treatments than the strong responses elicited by intense shock. Development of knowledge about the aversive properties of loud noise will allow not only verification of findings on shock maintained behavior but also the systematic exploration of interactions between aversive stimuli.

\section{References}

Azrin, N. H. Effects of punishment intensity during variable interval reinforcement. J. exp. Anal. Behav., 1960, 3, 123-142.

Barry, J. J., \& Harrison, J. M. Relation between stimulus intensity and strength of responding. Psychol. Rep., 1957, 3, 3-8.

Harrison, J. M., \& Abelson, R. M. The maintenance of behavior by the termination and onset of intense noise. J. exp. Anal. Behav., 1959, 2, 23-42.

Harrison, J. M., \& Tracy, W. H. Use of auditory stimuli to maintain lever pressing behavior. Science, 1955, 121, 373-374.

Holz, W. C., \& Azrin, N. H. Recovery during punishment by intense noise. Psychol. Rep., 1962, 11, 655-657.

Leitenberg, H. Conditioned acceleration and conditioned suppression in pigeons. J. exp. Anal. Behav., 1966, 9, 205-212.

\section{Note}

1. Part of a thesis submitted in partial fulfillment of requirements for the Master of Science degree. Deepest thanks are extended to Dr. R. A. Patton and Dr. D. C. Kruper for giving advice and making apparatus available. This study was supported by grant number MH 07227-03. 